\title{
COMMISSION 10: SOLAR ACTIVITY
}

\section{(ACTIVITE SOLAIRE)}

\section{PRESIDENT: Arnold O. Benz}

VICE-PRESIDENT: Donald B. Melrose

ORGANIZING COMMITTEE: G. Ai, N. Gopalswamy, R.A. Harrison,

A. Hood, B.V. Jackson, B.Z. Kozlovsky, G. Poletto, C.J. Schrijver,

K. Shibata, L. van Driel-Gesztelyi \& P. Venkatakrishnan

\section{Introduction}

The past three years have been a period of important progress in the field of solar activity that will have a permanent impact in the understanding of the Sun. The two fields of solar and stellar astrophysics are approaching each other by finding more and more questions of joint interest, including the evolution of solar activity in past and in future, the physics of activity, magnetic field build up and energy release.

Magnetic activity in cool stars such as the Sun is omnipresent in the outer layers and atmosphere. Its influence increases with radius and dominates in the corona. Commission 10 concentrates on the dynamic aspects of solar activity and in particular on the release of magnetic energy, particle acceleration, heating of the corona and origin of the solar wind. The following is a summary of progress in a selection of areas and we point out that it cannot be considered to be complete.

\section{Scientific Highlights}

Solar activity was very high in the triennium July 1999 - July 2002, reaching its 11-year peak in July 2000. The RHESSI satellite was launched, SOHO, TRACE and Yohkoh (until the end of 2001) continued to produce a rich harvest of data. In particular, we note the advances in exploring subsurface activity by acoustic imaging, coronal seismology in EUV lines, and continuous coronal variability indicating substantial mass and energy input in quiet regions.

\section{Surface Activity: Present and Past}

In the past few years, acoustic imaging of the photosphere has rapidly developed into a productive new field observing solar activity from an entirely new perspective. For the first time the structure of plasma and field below an active region was determined (Kosovichev et al. 2000), for the first time an active region was located (albeit a posteriori) before it emerged onto the surface, and activity on the backside of the Sun was imaged (Lindsey \& Braun 2000; Braun \& Lindsey 2001; Chou 2000).

Advances in imaging techniques of the surfaces of magnetically active stars other than the Sun are increasingly revealing stellar surface structure and dynamics that can be compared to solar observations and theory. There is now, for example, a consensus that polar and high-latitude starspots are common among the most active stars, and that these even occur on young, rapidly-rotating stars of solar spectral type (see, e.g., Granzer et al. 2000, for a discussion and a model). Such high-latitude activity poses interesting constraints either on models of the solar dynamo or on the formation and evolution of magnetic spots: there must either be a change in where most of the field emerges onto the surface as a 
function of stellar activity (e.g., Granzer et al. 2000), or spots must be able to form by flux coagulation well after emergence (Schrijver \& Title 2001).

Starspot maps (with starspot filling factors that can be as high as $70 \%$ in the most active stars) have been used to derive the stellar differential rotation at the surface (e.g., Collier Cameron 2000). Interestingly, most cool stars rotate such that the pole-equator lap time is rather similar to that of the Sun, even for stars with rotation periods that are almost 25 times shorter than that of the Sun. The meridional displacements of magnetic fields on these stars, on the other hand, still remain to be determined. These two stellar quantities are of direct importance to understand their nature on the Sun and their function in the solar dynamo.

An EUVE survey of cool stars including young solar analogs indicates a steep energy distribution of flares larger than $10^{32} \mathrm{erg}$ (Audard et al. 1999, 2000). The energy of these flares accounts for $10 \%$ of the $\mathrm{X}$-ray luminosity of the stellar coronae. The approximative power-law distribution has an index of $-2.0( \pm 0.2)$. Thus a considerable portion of the energy required to heat these coronae could be provided by flares. Nevertheless, the 'faint, young Sun paradox' to explain the presence of water on Earth and Mars cannot be interpreted by a large mass loss of the early solar wind, as deep radio observations of the young solar analog $\pi^{01} \mathrm{UMa}$ indicate (Gaidos et al. 2000).

The loss of mass and field leads to the formation of asterospheres, the stellar counterparts of our own heliosphere (Wood et al. 2001). Such observations, combined with modeling, allow a unique way to determine the mass loss of stars as inactive as the Sun, a parameter that is difficult even for the Sun itself because we can only sample the heliosphere with spacecraft that are few and far between.

One other side of the solar-stellar connection is the investigation of planetary systems other than our own and at different ages. Magnetic flaring in stellar analogs of the premain-sequence Sun in the Orion Nebula cluster has been observed in soft X-rays, suggesting a $10^{5}$-fold enhancement in energetic protons compared to contemporary levels. The flare proton flux is sufficient to produce the observed meteoritic abundances of several important short-lived radioactive isotopes in the disk of the early Sun (Feigelson et al. 2002).

\section{Fundamental Processes of the Quiet Solar Atmosphere}

\subsection{Coronal Heating and Transient Events}

The period covered by this report have seen a resurgence of interest in the quiet Sun. In particular, there are numerous efforts being made to understand the fundamental processes of the solar atmosphere through quiet Sun observations, in particular in the extreme-UV with the SOHO spacecraft, and in X-rays with Yohkoh. A number of small-scale globally distributed, quiet region event-types have been noted in recent years, such as explosive events (see Innes 2001) and blinkers (see Harrison et al. 1999) in the transition region (TR), and network flares (Krucker \& Benz 2000), and micro-events or nanoflares (review by Benz \& Krucker 2002) at coronal temperatures. These are all small-scale events detected as brightenings in EUV or soft X-ray radiation, or as transient Doppler shifts. The importance of such events for the identification of basic processes in the quiet Sun atmosphere has been recognized.

The most significant obstacle to understanding the influence of these events is observational, i.e. events detected at differing wavelengths, referring to different temperature regimes and different instruments. It is not clear whether transient events such as blinkers or explosive events observed at TR level are the low temperature counterpart of coronal events seen in soft X-rays (e.g. Winebarger et al. 2002b). Bewsher et al. (2002) focussed on blinkers, identifying a large number of these events and extending previous work, based on more limited sets of data (Harrison et al. 1999; Brković et al. 2000). However, no coronal counterpart of blinkers has been detected and the number of blinkers turns out to be significantly lower than the occurrence rate for coronal nanoflares as evaluated by Krucker \& Benz (1998). Another work, focused on explosive events, has been carried out 
by Teriaca et al. (2002), who showed that these events, although much more frequent than blinkers (Ryutova \& Tarbell 2000), still have no coronal counterpart. Nevertheless, the large majority of blinkers and explosive events occur over the network, which is claimed to be the primary energy source for the quiet corona (and fast wind as well, see Ryutova et al. 2001).

Different event-types may be related though, and there is a school of thought that many of these transient events are caused by the same physical processes. A relation between some brightenings in the transition region and corona has been demonstrated by cross-correlation of single pixels in time (Benz \& Krucker 1999). A 'unification' workshop was held in Paris in December 2001 aimed at identifying the different quiet Sun event-types when observational limitations are removed. The aim is to make a major advance in understanding the quiet Sun processes by combining various wavelengths.

Cell and network regions have been analyzed also to check for the presence and characteristics of brightenings that might be interpreted in the context of coronal heating by nanoflares events. Harra et al. (2000) pointed out the need for a separate analysis of cell and network regions, because the distributions of events have different energy power-law exponents in the two areas. This is an important parameter for any nanoflare heating theory, but its value is not definite yet. More studies have been published by Parnell \& Jupp (2000) and Aschwanden et al. (2000), discrepancies have been discussed by Benz \& Krucker (2002). Still, the extrapolation to events with energies lower than $\approx 10^{24} \mathrm{erg}$ is questionable, but vital for the theory (Winebarger et al. 2002a).

A number of nanoflare heating models for the quiet corona have been developed in the last few years: Mitra-Kraev \& Benz (2001) have demonstrated that models exist that fit all observed constraints, including the observed energy distribution of events, baseline energy requirement, power spectrum, and emission measure variation in time. Georgoulis et al. (2001) developed models based on the Self Organized Criticality idea from the observed power-law distribution of the energy flux of nanoflares. Cellular automata techniques have been used by Krasnoselskikh et al. (2002) in a model where the authors examine how characteristics of the dissipated energy flow depend upon properties of the magnetic field source. At the higher coronal level, Mandrini et al. (2000) have tested coronal heating models deriving scaling laws for magnetic and plasma parameters in quasi-static soft X-ray loops. Their results imply that models based on the gradual stressing of the magnetic field are in generally better agreement with observational constraints than wave heating models. If the photospheric field is considered to be concentrated at the base of thin flux tubes, soft X-ray coronal observations are in the best agreement with models invoking stochastic build up of energy, current layers and MHD turbulence, all models implying energy release in highly episodic events (microflares or nanoflares) that may occur all along the coronal loop.

\subsection{Solar Wind Acceleration}

The SOHO spectroscopic capability, using the CDS and SUMER instruments, has also fueled a new interest in studies of wave activity off the limb. The aim is to detect evidence for wave propagation and dissipation through the analysis of UV/EUV emission lines. Most of this work has involved the observation of emission line broadening with altitude above coronal holes (e.g. Banerjee et al. 2000 and Doscheck et al. 2001). This is consistent with the detection of Alfvén wave propagation along open field lines. However, similar studies in the closed structures above the equatorial quiet Sun have shown evidence for emission line narrowing (Harrison et al. 2002) which has been interpreted as evidence for wave dissipation in the closed loops above the limb.

In solar X-ray images, we easily distinguish quiet from coronal hole areas, because, at a temperature of about $1.5 \cdot 10^{6} \mathrm{~K}$, the coronal hole $(\mathrm{CH})$ plasma emission is about one order of magnitude weaker than in the quiet Sun (QS). However, images of the quiet Sun in lines forming at progressively lower temperatures show less and less difference between the QS and CH areas. Frequency distribution of line intensities, widths and Doppler shifts 
turn out to depend on whether data refer to coronal holes or to quiet regions. This kind of analysis has been used by many authors (see, e.g. Peter \& Judge 1999; Stucki et al. 2000, 2002; Raju et al. 2000, Pauluhn et al. 2000) in an attempt to identify the level and/or temperature in the solar atmosphere, where the solar wind originates.

These works face two problems, namely (i) the identification of the rest wavelengths of the lines that are analyzed and (ii) the structure of the network in high temperature lines. As a consequence, outflow speeds are often expressed in terms of differences between quiet Sun and coronal holes, rather than in absolute units, and the positions of the measured outflows are expressed in terms of the position relative to the network, as seen in lines which form at lower temperatures than those used in the outflow analysis. With these caveats in mind, there is now a general consensus on coronal hole lines being blue-shifted, relative to the quiet Sun, at temperatures above $10^{5} \mathrm{~K}$. The interpretation of this result in terms of nascent solar wind is attractive, but needs to be substantiated by further analyses, being the difference between blue-shift in $\mathrm{CH}$ and QS regions rather small. However, line widths and Doppler shifts can be fitted into a unique scenario that encompasses network and internetwork regions, from the transition region out to the corona, as proposed by Peter $(2000,2001)$. Furthermore, Brown et al. (2000) have noted that an order of magnitude more chromospheric material is evaporated in coronal micro-events (nanoflares) than observed in the solar wind. This may indicate that mass injection is not a continuous process, but occurs impulsively.

Where in coronal holes, solar wind accelerates, is still not definitely settled. Hassler et al. (1999) claimed that the highest blue shifts, in Ne VIII, occur at junctions of chromospheric network lanes. Whether these are to be identified as the main sources of the fast solar wind, though, can be established only after comparing the value of the mass flux emanating from these areas with the mass flux measured in situ. An attempt in this direction has been made by Landi et al. (1999) and Wilhelm et al. (2000).

\section{Coronal Seismology of Active Region Loops}

The high spatial and temporal resolution of present spacecrafts, such as SOHO and TRACE, has produced increasing observational evidence for both longitudinal and transversal coronal oscillations. De Moortel et al. (2000) and Robbrecht et al. (2001) reported oscillations propagating along magnetic loops. Transversal, flare excited oscillations have been used by Schrijver et al. (2002) and Aschwanden et al. (2002) to study decay time and Alfvén velocity. Furthermore, the 3 and 5 minute period oscillations have been detected in coronal loops (De Moortel et al. 2002), indicating that some longitudinal oscillations are not flaredriven, but most likely caused by a chromospheric or photospheric driver exciting the loop footpoints. This result suggests that some oscillations from underlying layers may propagate into the corona and there appear as magneto-acoustic waves.

\section{Flares and Particle Acceleration}

\subsection{White-light Flares}

During the last years progress has been made in understanding the cause of continuum enhancements in the so-called white-light flares (WLFs). Based on the analysis of Yohkoh/SXT and HXT images and using image de-convolution, Sylwester \& Sylwester (2000) found that SXR, HXR and white-light flare brightenings are not co-spatial and that they may be related to different plasma volumes at any instant. This finding does not corroborate with the idea that WLFs are caused by non-thermal electron beams and that SXR loops are filled via chromospheric evaporation from the HXR/WLF footpoints. Results using observations in other wavelength domains agreed with the above conclusion. WLF kernels usually correspond to the brightest portion of $\mathrm{H} \alpha$ kernels, but not always. Using the $\mathrm{H} \alpha$ line intensity as a constraint, Gan, Hénoux \& Fang (2000) concluded that neither chromospheric condensations, nor non-thermal effects alone can explain the continuum enhancements in WLFs. 
They proposed that the latter is indirectly produced by chromospheric condensation and the non-thermal effects via radiative heating of the deeper photospheric layers. The latter is in good agreement with the results of Liu, Ding, \& Fang (2001), who, based on H $\alpha$, CaII and $7.58 \mathrm{GHz}$ microwave observations of a WLF concluded that a non-thermal electron beam cannot directly produce continuum emission. Heating in the lower atmosphere resulting from the radiative back-warming may lead to the formation of continuum enhancement.

\subsection{On the Role of Magnetic Shear and Emerging Flux in the Flare Process}

There is a consensus that the energy released in flares must come from free energy of the magnetic field. Providing unambiguous evidence for this has not been an easy task, however. $\mathrm{Li}$ et al. $(2000 \mathrm{a}, 2000 \mathrm{~b})$ investigated the role of magnetic shear in ten flares using vector magnetograms, $\mathrm{H} \alpha$ and SXR images. Five of the flares were observed in active regions with low, and five others in regions with high magnetic shear. These authors found that although flare-related shear changes on the neutral line were small $\left(2^{\circ}-4^{\circ}\right)$ and the association of these changes with the flares was not conclusive, the average shear in the flaring areas of the flares associated with high shear decreased significantly after the flares. Flux emergence and the interaction between new and pre-existing flux appeared to play an important role in all the studied flare cases. Ishii et al. (2000), examining variations in the $\mathrm{H} \alpha$ fine structures and proper motions of sunspots, provided evidence that emergence of a twisted flux bundle may play a crucial role in the flare productivity of an active region, providing an injection of free magnetic energy for the active region.

\subsection{Studies of the 14 July 2000 Flare}

One of the highlights of the past three years was the broad multi-wavelength observational

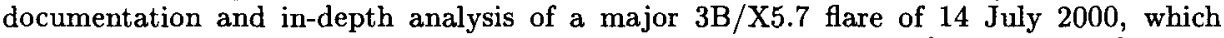
occurred close to the central meridian. The filament eruption in this $\delta$-spot region, followed by the formation of a curved arcade with some 100 post-flare loops, which brightened up in a sequential manner, from highly-sheared low-lying to less-sheared higher-lying loops, appeared spectacular in a TRACE movie. The event produced all the solar, interplanetary and terrestrial signatures expected from a major eruptive flare and up to date, its study led to the publication of nearly 50 journal articles, many of which appeared in a special 'Bastille Day flare' volume of Solar Physics (2001, vol. 204).

\subsection{Particle Acceleration}

Data from spacecraft (WIND, SOHO, ACE, Yohkoh, RHESSI) are providing increasingly detailed knowledge of the composition and spectra of solar energetic particles (SEPs), and on the various correlations between different species of SEPs and between SEPs and other solar phenomena, notably CMEs and type II radio bursts. The anomalous overabundance of ${ }^{3} \mathrm{He}$ remains an important discriminator for SEP events. Information on other anomalies are becoming available from detailed studies, including enhancements of ions with $34 \leq Z \leq 40$ and $50 \leq Z \leq 56$ by $\sim 100$ and $\sim 1000$, respectively, relative to $\mathrm{O}$ (Reames, $\mathrm{Ng}$ and Tylka 2001), and of specific isotopes, such as ${ }^{14} \mathrm{~N}$ and ${ }^{28} \mathrm{Si}$ in ${ }^{3} \mathrm{He}$-rich events (Mason, Mazur and Dwyer 2002). Such details provide challenges to the standard interpretation for such anomalies in terms of pre-acceleration by self-generated cyclotron waves.

A new interpretation for the enhanced acceleration of ${ }^{3} \mathrm{He}$ has been proposed by Paesold et al. (2002) based on the previously proposed electron firehose instability of an anisotropic electron distribution enhanced along the direction of the magnetic field. Such a distribution is predicted by all proposed acceleration processes (Paesold \& Benz 1999; Li \& Habbal 2000). It causes left circularly polarized electromagnetic waves to grow which preferentially interact with ${ }^{3} \mathrm{He}$ in gyro-resonance. In this model, the acceleration of ${ }^{3} \mathrm{He}$ becomes a genuine ingredient of electron acceleration. The instability also provides a means to keep the electron population close to isotropic, a necessary requirement e.g. for acceleration 
by transit-time damping of magnetoacoustic waves. The electron firehose interpretation provides a testable link between ${ }^{3} \mathrm{He}$ and electron acceleration and could be an important step toward the understanding of flare particle acceleration.

Spacecraft data also yielded further detailed information on the acceleration of electrons. Evidence for loop-top heating in solar flares tends to favor a model based on heating and acceleration during reconnection (Craig \& Litvinenko 2002). However, shock acceleration is invoked for mildly relativistic electrons (Klassen et al. 2002). Rich- and poor-SEP events show a correlation with the associated CMEs (Gopalswamy et al. 2002a). Krucker $\& \operatorname{Lin}(2000)$ have found delayed SEP events also in impulsive flares and demonstrated evidence for acceleration at altitudes consistent with CMEs or travelling coronal disturbances. Prompt electrons in SEP events have been traced back to the corona by Benz et al. (2001). The highest frequencies of radio emissions found were mostly of metric type III bursts and in some cases metric narrowband spikes.

Narrowband radio spikes at meter waves have been found to be closely associated in space with electron beams manifest by their type III radio emission (Paesold et al. 2001). At decimeter waves the narrowband spikes were found at high coronal altitude by Benz et al. (2002). Both observations indicate the presence of radio sources near or in the coronal acceleration region. However, the radio emission process of narrowband spike is as unclear as ever.

Rank et al. (2001) have investigated extended gamma-ray emission in large flares, indicating that the processes in the extended phase differ from those during the impulsive phase of flares but require continuous acceleration. Long-term trapping is unlikely to explain the pion-decay radiation, caused by high-energy protons, that was not detected before the onset of the extended emission phase. In preparation for RHESSI observations, Kozlovsky et al. (2002) updated cross sections for the production of gamma-ray lines from the impact of accelerated ions on the solar atmosphere and Hua et al. (2002) detailed the production kinematics and cross sections for neutrons that produce the $2.223 \mathrm{MeV}$ capture line that dominates most gamma-ray flare spectra. Using moderate resolution, Share et al. (2002) studied the Doppler shifts of the $4.44 \mathrm{MeV}$ de-excitation line from ${ }^{12} \mathrm{C}$ as a function of flare heliocentric angle. They found that the shifts are consistent with a predominately downward isotropic distribution of interacting particles. Such a distribution occurs for particles that undergo significant pitch-angle scattering by wave turbulence in coronal magnetic loops after acceleration.

\section{First Determinations of Magnetic Helicity}

After a decade of work on current helicity which produced several important results, e.g. the discovery of the hemispheric helicity rules, several groups succeeded to develop methods to evaluate magnetic helicity from observations.

The method to determine observationally the rate of magnetic helicity injection through the solar surface via horizontal photospheric motions of field line foot-points, other than differential rotation, was first discussed by Chae (2001). Following this, Chae et al. (2001) studied the contribution of these motions to the formation of a filament and found that they injected $10^{42} \mathrm{Mx}^{2}$ of magnetic helicity into the coronal fields. This is of the order of the helicity shed in magnetic clouds (DeVore 2000). On the longer term, Démoulin et al. (2002) and Green et al. (2002) studied the helicity evolution of two active regions (NOAA 7978 \& 8100 , respectively). They estimated how much helicity is shed by CMEs during the entire existence of the two ARs. After correction for data gaps and far-side locations, the two ARs were found to produce 32 and 65 CMEs, which carried away a sum of helicity in the order of $10^{43}-10^{44} \mathrm{Mx}^{2}$. The authors concluded that the main source of coronal magnetic helicity must be the inherent twist of the emerging flux tubes, since during the same period the differential rotation generated only a fraction of this. The sustained CME productivity of ARs into their decay phase suggests that helicity is continuously supplied to the corona from the sub-photospheric layers either by continued flux emergence, or via torsional Alfvén waves. Nindos \& Zhang (2002) made a study over a shorter time period of 
the CME activity and helicity injection by shear motions in active region 9165 . They also found that the CMEs originating in the AR remove a factor of 8-64 more helicity than the helicity injected by horizontal shearing motions. Even though the computed helicity change due to horizontal shearing motions, in this case, is probably the largest ever reported.

The overall picture emerging from these works shows that theoretical models need to include the emergence of magnetic field twisted deep within the Sun and its interaction with the pre-existing corona, and not to rely on photospheric differential rotation to eruptively destabilize the magnetic environment in order to produce CMEs.

\section{Coronal Mass Ejections, Space Weather and Solar Terrestrial Relations}

The coronal mass ejection (CME) has become one of the hottest topics in the study of solar activity because of the extensive influence CMEs have on the inner heliosphere (Schwenn 2000; Low 2001). A special section in the Journal of Geophysical Research, devoted to the global picture of CMEs was published (Gopalswamy 2000).

Efforts have continued, to understand the detection of EUV/X-ray dimming in the low corona underneath CMEs (Thompson et al. 2000b; Harrison and Lyons 2000; Gopalswamy \& Thompson 2000; Wang et al. 2000; Webb et al. 2000; Wu et al. 2001; Harra \& Sterling 2001). Spectroscopic observations confirm that the dimming is due to mass-loss in the low corona, and the estimated missing mass is equivalent to that of the overlying CME. These observations are being used to explore the CME source regions and the onset process as never before. It is notoriously difficult to identify the CME source region even with coronal imaging instruments, and the dimming technique is a major step in this work. Coronal dimming in EUV and X-rays associated with solar eruptions has become a common tool to identify the region of eruption. Identification of solar sources for halo CMEs is essential to decide whether the CMEs are directed towards or away from Earth.

Another feature closely linked with the CME onset is the detection of coronal (EIT) waves, where significant disturbances cross the corona at hundreds of $\mathrm{km} / \mathrm{s}$ in association with a CME onset and, often, a flare. The debate on the significance of this continues. A dedicated session at the International Solar Cycle Studies (ISCS) meeting in Longmont, USA, in June 2001, highlighted the cause and effect problem, with the general conclusion that there was something of the 'big flare syndrome' with major events including all components (flare, CME, EIT wave etc...) and other events occurring with only a subset of the components. This being the case, the EIT wave and CME are associated but one must be careful in drawing any conclusions about cause and effect.

\subsection{Coronal Mass Ejections, Shock Waves and EUV Transients}

CMEs drive magnetohydrodynamic shocks, which in turn accelerate electrons and ions. CMEs are also closely related to flares. In the following, progress made towards understanding the complex relationship between these phenomena is summarized.

Major progress in understanding the properties of CMEs is largely due to the Large Angle and Spectrometric Coronagraph (LASCO) on board the SOHO spacecraft. LASCO has observed more than 5000 CMEs since its launch in 1995 until the end of 2001. An initial statistical study of the CMEs observed until the end of June 1998 were performed by St. Cyr et al. (2000). A complete catalog of the CMEs and their measured properties is available on line (http://cdaw.gsfc.nasa.gov/CME_list). The Wind spacecraft launched about a year before SOHO carried the WAVES experiment which detects radio emission due to CME-driven shocks. The SOHO-Wind combination has been extremely useful in studying the solar origin of interplanetary disturbances.

Inner coronal imaging from the Extreme-ultraviolet Imaging Telescope (EIT) on board SOHO has helped in identifying the solar sources of CMEs (Webb 2000; Hudson \& Cliver 2001; Gopalswamy et al. 2000a,b, 2001a,b). A subset of these waves known as "brow waves" because of their morphological feature resembling an eyebrow, may be the manifestation 
of shocks (Thompson et al. 2000b; Gopalswamy \& Thompson 2000; Klassen et al. 2000; Biesecker et al. 2002). In one case, metric type II burst imaged by the Nançay radioheliograph was found to be spatially and temporally associated with a brow wave (Gopalswamy et al. 2000c). The EIT waves (at least the brow type) may be the coronal counterparts of $\mathrm{H} \alpha$ Moreton waves (Thompson et al. 2000a; Warmuth et al. 2001; Pohjolainen et al. 2001), although Eto et al. (2002) showed evidence that the classical EIT wave is different from the $\mathrm{H} \alpha$ Moreton wave. Attempts have also been made to identify the $\mathrm{X}$-ray and microwave counterpart of EIT waves and how the X-ray wave transient may be related to the H-alpha Moreton waves (Narukage et al. 2002; Khan \& Aurass 2002; Aurass et al. 2002).

\subsection{CMEs Association with Metric and Interplanetary Type II Bursts}

The age-old controversy regarding the relationship between coronal and interplanetary type II bursts has been revisited recently because the WAVES data provide observations in the near-Sun interplanetary medium, adjacent to the corona probed by ground-based instruments. Reiner et al. (2001) found a close relationship between the speeds of IP shocks inferred from DH type II bursts and CME speeds, while there is no such correlation between shock speeds inferred from metric type II bursts and CME speeds. On the other hand, Vrsnak (2001) found a positive correlation between shock speeds inferred from metric type II bursts and flare importance and hence concluded that the metric type II bursts are due to flare blast waves. Gopalswamy et al. (2001a) and Gopalswamy \& Kaiser (2002) computed the radial profile of the magnetosonic speed in the corona, which shows a broad peak near the $3 \mathrm{Rs}$. Since it is difficult to drive a shock around $3 \mathrm{Rs}$, the same disturbance (CME) may drive metric and IP shocks (on either side of this peak) propagating under two different ambient characteristics.

Apart from the detection of CME-driven shocks from LASCO observations (Sheeley et al. 2000), the Wind/WAVES experiment has provided new information on CME-driven shocks. By observing type II bursts in the 1-14 $\mathrm{MHz}$ range, it was found that the CMEs responsible for the shocks are faster and wider than the average CMEs (Gopalswamy et al. 2001b). Spectacular continuum-like radio emissions immediately following type II bursts were recently discovered (Gopalswamy al. 2001c), the origin of which can be traced to colliding CMEs. Radio emission was also found without a preceding type II burst suggesting the possibility of electron acceleration due to colliding CMEs in the outer corona and beyond (Gopalswamy et al. 2002a).

On the theory side, a new semi-analytic theory for type II solar radio bursts was recently proposed by Knock et al. (2001), which combines electron reflection and energization at the shock, development of beam distributions in the foreshock by time-of-flight effects, stochastic growth of Langmuir waves, and generation of radiation by specific nonlinear Langmuir wave processes. Savoini \& Lembége (2001) performed 2-D simulations of a curved shock front and investigated the influence of the curvature geometry and different spatial scales on electron acceleration mechanism.

\subsection{CME Initiation and Emerging Flux}

Emerging flux continues to be pursued as a possible trigger to CMEs and flares (Nitta \& Hudson 2001; Srivastava et al. 2000). Photospheric magnetic field changes associated with CMEs is not readily identified, although Lara et al. (2000) have shown clear changes over small regions within the overall region of eruption. Changes in magnetic flux seem to take place over time scales much longer than those over which the CMEs are initiated (Subramanian \& Dere 2001). Although bipolar regions are not thought to be good regions for CME production, Moore et al. (2001) have shown that internal reconnection of the sheared and twisted (in the shape of a sigmoid) core fields in single bipoles is essential for the eruptions of coronal mass ejections (see Sterling \& Moore, 2001 for a detailed discussion on internal and external reconnections in eruption regions). Zhang et al. (2001) investigated the relationship between flares and CMEs and concluded that while CMEs clearly precede 
flares, the latter can influence the CME acceleration profiles. Chen \& Shibata (2000) have developed a CME model triggered by emerging flux using 2D-MHD numerical simulations.

\subsection{CMEs and Prominence Eruptions}

Eruptive prominences form the core of CMEs and represent one of the early manifestations of the CME phenomenon. Since it is well known that all prominences can be divided into inverse and normal polarity types, one might expect that CMEs somehow reflect the type of the prominence. In fact, Low \& Zhang (2002) have attempted to relate the flux-rope CMEs to the initial states of prominences. Part or whole of eruptive prominences are known to fall back after reaching a maximum height (Gilbert et al. 2000). So one would expect infalling prominence material from coronagraph observations, but rarely observed. Recently, Wang \& Sheeley (2002) reported several "core fallback" events using SOHO/LASCO data and suggested that the dynamical behavior of the CME core may be determined in part by momentum exchanges with the ambient medium. This momentum coupling is close to the Sun as opposed to the overall interaction between the whole CME and the interplanetary medium between the Sun and Earth (Gopalswamy et al. 2001d). Radio observations have become an important source of data on eruptive prominences, indicating a good correlation between CMEs and prominence eruptions (Hori \& Culhane 2002). Substructures of CMEs can also be imaged using thermal (Marque et al. 2002) and nonthermal emissions (Bastian et al. 2001).

Measurement of properties such as the width and speed of Earth-directed CMEs is crucial from the point of view of space weather. SOHO's Ultraviolet Coronagraph Spectrometer (UVCS) has been useful in obtaining the line of sight velocity of CME substructures so that by combining with LASCO observations, one can get some information on the 3-D properties of CMEs (Raymond et al. 2000; Ciaravella et al. 2000, 2001; Akmal et al. 2001).

\subsection{CMEs and Solar Energetic Particles}

Large, gradual solar energetic particle (SEP) events are known to be closely associated with CMEs. This is taken as evidence for SEP acceleration by the CME-driven shocks (Kallenrode 2001). Consistent with this idea, Kahler et al. (2001) found that post-eruption arcades (whether in or outside active regions) were not sources of escaping gradual SEPs. However, Kahler et al. (2001) also note that many impulsive SEP events are accompanied by narrow CMEs. Thus, CMEs seem to be an important ingredient for both impulsive and gradual SEP events (Laitinen et al. 2000; Torsti et al. 2001). The traditional classification of SEPs as impulsive and gradual events, caused by flares and CMEs, respectively, seems to be more and more blurred as new observations indicate.

A. O. Benz

President of the Commission

\section{References}

Akmal, A., Raymond, J. C., Vourlidas, A., Thompson, B., Ciaravella, A., Ko, Y.-K., Uzzo, M., \& Wu, R., 2001, , ApJ, 553, 922

Aschwanden, M.J., et al., 2000, ApJ, 535, 1047

Aschwanden, M.J., De Pontieu, B., Schrijver, C.J., \& Title, A.M., 2002, Solar Phys., 206, 99

Audard, M., Güdel, M., \& Guinan, E.F., 1999, ApJ, 513, L53

Audard, M., Drake, J.J., Kashyap, V.L., 2000, ApJ, 541, 396

Aurass, H., Shibasaki, K., Reiner, M. \& Karlick, M., 2002, , ApJ, 567, 610

Banerjee, D., Teriaca, L., Doyle, J.G., \& Lemaire, P., 2000, Solar Phys. 194, 43

Bastian, T. S., Pick, M., Kerdraon, A., Maia, D., \& Vourlidas, A., 2001, ApJ, 558, L65 
Benz, A.O. \& Krucker, S., 1999, A\&A, 341, 286

Benz, A.O., Lin, R.P., Sheiner, O.A., Krucker, S., \& Fainberg, J., 2001, Solar Phys., 203, 131

Benz, A.O., Saint-Hilaire, P., \& Vilmer, N., 2002, A\&A, 383, 678

Benz, A.O. \& Krucker, S., 2002, ApJ, 568, 413

Bewsher, D., Parnell, C.E., \& Harrison, R.A., 2002, Solar Phys., 206, 21

Biesecker, D.A., Myers, D.C., Thompson, B.J., Hammer, D.M., \& Vourlidas, A., 2002, ApJ, 569,1009

Braun, D. C., \& Lindsey, C., 2001, ApJ, 560, L189

Brković, A., Rüedi, I., Solanki, S.K., Fludra, A., Harrison, R.A., Huber, M.C.E., Stenflo, J.O., \& Stucki, K., 2000, A\&A, 353, 1083

Brown, J.C., Krucker, S., Güdel, M., \& Benz, A.O., 2000, A\&A, 359, 1185

Chae, J., 2001, ApJ, 560, 95

Chae, J., Wang, H., Qiu, J., Goode, P.R., Strous, L., Yun, H.S., 2001, ApJ, 560, 476

Chen, P. F. \& Shibata, K., 2000, ApJ, 545, 524

Ciaravella, A., et al., 2000, ApJ, 529, 575

Ciaravella, A., Raymond, J. C., Reale, F., Strachan, L., \& Peres, G., 2001, ApJ, 557, 351

Collier Cameron, A., 2000, IAU Symposium 203 on 'Spectroscopy of Active Stars and Stellar Prominences', 317

Craig, I.D.J, \& Litvinenko, Y.E., 2002, ApJ, 570, 387

De Moortel, I., Ireland, J., \& Walsh, R.W., 2000, A\&A, 355, L23

De Moortel, I., Ireland, J., Hood, A.W., \& Walsh, R.W., 2002, A\&A, 387, L13

Démoulin, P., Mandrini, C.H., van Driel-Gesztelyi, L., Thompson B., Plunkett S., Kovari Zs., Aulanier, G. \& Young, A., 2002, A\&A, 382, 650

Doschek, G., Feldman, U., Laming, J.M., Schühle, U., \& Wilhelm, K., 2001, ApJ, 546, 559

DeVore, R., 2000, ApJ, 539, 944

Eto, S. et al., 2002, PASJ, 54, 481

Feigelson, E.D., Garmire, G.P., \& Pravdo, S.H., 2002, ApJ, 572, 335

Filippov, B. P., Gopalswamy, N., \& Lozhechkin, 2001, Solar Phys., 203, 119

Gaidos, E.J., Güdel M. \& Blake, G.A., 2000, Geophys. Res. Let., 27, 501

Gan, W.Q., Hénoux , J.-C., \& Fang, C., 2000, A\&A, 354, 691

Georgoulis, M.K., Vilmer, N., \& Crosby, N.B., 2001, A\&A, 367, 326

Gibson, S. E., \& Low, B. C., 2000, J. Geophys. Res., 105, 18187

Gilbert, H., Holzer, T. E., Burkepile, J, T. \& Hundhausen, A. J., 2000, ApJ, 537, 503

Gopalswamy, N., 2000, AGU monograph 119, p. 123

Gopalswamy, N., \& Thompson, B. J., 2000, JATP, 62, 1457

Gopalswamy, N., Lara, A., Lepping, R. L., Kaiser, M. L., Berdichevsky, D., \& St. Cyr, O. C., 2000a, Geophy. Res. Lett., 27, 145

Gopalswamy, N., Kaiser, Thompson, B. J., Burlaga, L., Szabo, A., Vourlidas, A., Lara, A., Yashiro, S., \& Bougeret, J.-L., 2000b, Geophy. Res. Lett., 27, 1427

Gopalswamy, N., Kaiser, M. L., Sato, J., \& Pick, M., 2000c, ASP Conference Series, Vol. 206, Edited by R. Ramaty \& N. Mandzhavidze, p.351

Gopalswamy, N., Lara, A. Kaiser, M. L., \& Bougeret, J.-L., 2001a, J. Geophys. Res., 106, 25261

Gopalswamy, N., Yashiro, S., Kaiser, M. L., Howard, R. A. \& Bougeret, J.-L., 2001b, J. Geophys. Res., 106, 29219

Gopalswamy, N., Yashiro, S., Kaiser, M. L., Howard, R. A., \& Bougeret, J.-L., 2001c, ApJ, 548, L91 
Gopalswamy, N., St. Cyr, O. C., Kaiser, M. L., \& Yashiro, S., 2001d, Solar Phys., 203, 149 Gopalswamy, N., Lara, A., Yashiro, S., Kaiser, M. L., \& Howard, R. A., 2001d, J. Geophys. Res., 106, 29207

Gopalswamy, N., Yashiro, S., Kaiser, M. L., Howard, R. A. \& Bougeret, J.-L., 2002a, Geophys. Res. Lett., 29(8), 10

Gopalswamy, N., \& Kaiser, M. L. 2002a, Adv. Space Res., 29(3), 307

Granzer, T., Schüssler, M. Caligari, P. \& Strassmeier, K.G., 2000, A\&A, 355, 1087

Green, L.M., López Fuentes, M.C, Mandrini C.H., Démoulin P., van Driel-Gesztelyi L., \& Culhane, J.L., 2002, Solar Phys., 208, 43

Harra, L.K., Gallagher, P.T., \& Phillips, K.J.H., 2000, A\&A, 362, 371

Harra,L.K. \& Sterling, A.C., 2001, ApJ, 561, L215

Harrison, R.A., Lang, J., Brooks, D.H., \& Innes, D.E., 1999, A\&A, 351, 1115

Harrison, R.A. \& Lyons, M., 2000, A\&A, 358, 1097

Harrison, R.A., Hood, A.W., \& Pike, C.D., 2002, A\&A, 392, 319

Hassler, D.,M., Dammasch, I.E., Lemaire, P., et al., 1999, Science, 283, 810

Hori, K. \& Culhane, L., 2002, A\&A, 382, 666

Hua, X.-M., Kozlovsky, B., Lingenfelter, R. E., Ramaty, R., \& Stupp, A., 2002, Ap. J. Supp., 140, 563

Hudson, H. S. \& Cliver, E. W., 2001, J. Geophys. Res., 106, 25199

Innes, D.E., 2001, A\&A, 378, 1067

Ishii, T., Kurokawa, H., \& Takeuchi, T., 2000, PASJ, 52, 337

Kahler, S. W., McAllister, A. H., \& Cane, H. V., 2000, ApJ, 533, 1063

Kahler, S. W., Reames, D. V., \& Sheeley, N. R., Jr., 2001, ApJ, 562, 558

Kallenrode, M., 2001, J. Geophys. Res., 106, 24989

Khan, J. I. \& Aurass, H., 2002, A\&A, 383, 1018

Klassen, A., Aurass, H., Mann, G., \& Thompson, B. J., 2000, A\&A, 141,357

Klassen, A., et al., 2002, A\&A, 385, 1078

Knock, S. A., Cairns, I. H., Robinson, P. A. \& Kuncic, Z., 2001, J. Geophys. Res., 106, 25041

Kosovichev, A. G., et al., 2000, Solar Phys., 192, 159

Kozlovsky, B., Murphy, R. J., \& Ramaty, R., 2002, Ap. J. Supp., 141, 523

Krasnoselskikh, V., Podlachikova, O., Lefebvre, B., \& Vilmer, N., 2002, A\&A, 382, 699

Krucker, S., \& Benz, A., 1998, ApJ, 501, L213

Krucker, S., \& Benz, A., 2000, Solar Phys., 191, 341

Krucker, S., \& Lin, R.P., 2000, ApJ, 542, 61

Landi, E., Mullan, D., Poletto, G., \& Wilhelm, K., 1999, in "Proceedings of SOHO 8", p. 417

Laitinen, T., Klein, K.-L., Kocharov, L., Torsti, J., Trottet, G., Bothmer, V., Kaiser, M. L., Rank, G., \& Reiner, M. J., 2000, A\&A, 360, 729

Lara, A., Gopalswamy, N., \& DeForest, C. 2000, Geophys. Res. Lett., 27, 1435

Li, X. \& Habbal, S.R., 2000, J. Geophys. Res., 105, 27377

Li, H., Sakurai, T., Ichimoto, K., \& Ueno, S., 2000a, PASJ, 52, 465

Li, H., Sakurai, T., Ichimoto, K., \& Ueno, S., 2000b, PASJ, 52, 483

Lindsey, C., \& Braun, D.C., 2000, Solar Phys., 192, 261

Liu, Y., Ding, M.D., \& Fang, C., 2001, ApJ, 563, L169

Low, B.C., 2001, J. Geophys. Res., 106, 25141

Low, B. C. \& Zhang, M., 2002, ApJ, 563, L53 
Mandrini, C.H., Démoulin, P., Klimchuk, J.A., 2000, ApJ, 530, 999

Marqué, Ch., Lantos, P., \& Delaboudinière, J. P., 2002, A\&A, 387, 317

Mason, G.M., Mazur, J.E., \& Dwyer, J.R., 2002, ApJ, 565, L51

Mitra-Kraev, U., \& Benz, A.O., 2001, A\&A, 373, 318

Moore, R.L., Sterling, A.C., Hudson, H.S., \& Lemen, J.R., 2001, ApJ, 552, 833

Narukage et al. 2002, ApJ, 572, L109

Nindos, A., \& Zhang, H., 2002, ApJ, 573, 133

Nitta, N., \& Hudson, H., 2001, Geophys. Res. Lett., 28, 3801

Paesold, G., \& Benz, A.O., 1999, A\&A, 351, 741

Paesold, G., Kallenbach, R.,\& Benz, A.O. 2002, ApJ, in press

Paesold, G., Benz, A.O., Klein, K.-L., \& Vilmer, N., 2001, A\&A, 371, 333

Parnell, C.E., \& Jupp, P.E., 2000, ApJ, 529, 554

Patsourakos, S., \& Vial, J.-C., 2002, A\&A, 385, 1073

Pauluhn, A., Solanki, S.K., Rüedi, I., Landi, E., \& Schühle, U., 2000, A\&A, 362, 737

Peter, H., \& Judge, P.G., 1999, ApJ, 522, 1148

Peter, H., 2000, A\&A, 360, 761

Peter, H., 2001, A\&A, 374, 1108

Pohjolainen, S., Maia, D., Pick, M., Vilmer, N., Khan, J. I., Otruba, W., Warmuth, A., Benz, A. O., Alissandrakis, C., \& Thompson, B. J., 2001, ApJ, 556, 421

Raju, K.P., Sakurai, T., Ichimoto, K., \& Singh, J., 2000, ApJ, 543, 1044

Rank, G., Ryan, J., Debrunner, H., McConnell, M., \& Schönfelder, V., 2001, A\&A, 378, 1046

Raymond, J. C., Thompson, B. J., St. Cyr, O. C., Gopalswamy, N., Kahler, S., Kaiser, M., Lara, A., Ciaravella, A., Romoli, M., \& O'Neal, R., 2000, Geophys. Res. Lett., 27, 1439

Reames, D. V., Ng, C. K., \& Tylka, A. J., 2001, ApJ, 548, L233

Reiner, M. J., Kaiser, M. L., Gopalswamy, N., Aurass, H., Mann, G., Vourlidas, A., \& Maksimovic, M., 2001, J. Geophys. Res., 106, 25279

Robbrecht et al. (2001)

Ryutova, M. P., \& Tarbell, T. D., 2000, ApJ, 541, L29

Ryutova, M. P., Habbal, S.R., Woo, R., \& Tarbell, T. D., 2001, Solar Phys., 200, 213

Savoini, P. \& Lembege, B., 2001, J. Geophys. Res., 106, 12975

Schwenn, R., 2000, Adv. Space Res., 26(1), 43

Schrijver, C. J., \& Title, A. M., 2001, ApJ, 551, 1099

Schrijver, C.J., Aschwanden, M.J., \& Title, A.M., 2002, Solar Phys., 206, 69

Share, G. H., Murphy, R. J., Kiener, J., \& de Sereville,N., 2002, ApJ, 573, 464

Sheeley, N., Hakkala, W. N. \& Wang, Y.-M., 2000, J. Geophys. Res., 105, 5081

Solar Physics, 2001, Topical issue on the '2000 Bastille day flare event', 204, 1-436.

Srivastava, N., Schwenn, R., Inhester, B., Martin, S. F., \& Hanaoka, Y., 2000, ApJ, 534, 468

St. Cyr, et al., 2001, J. Geophys. Res., 105, 18169

Sterling, A., \& Moore, R., 2001, J. Geophys. Res., 106, 25227

Stucki, K., et al., 2000, A\&A, 363, 1145

Stucki, K, Solanki, S.K., Pike, C.D., Schühle, U., Rüedi, I., Pauluhn, A., \& Brković, A., 2002, A\&A, 381, 653

Subramanian, P., \& Dere, K. P., 2001, ApJ, 561, 372

Sylwester, B. \& Sylwester, J., 2000, Solar Phys., 194, 305 
Teriaca, L., Madjarska, M.S., \& Doyle, J.G., 2002, A\&A, in press

Title, A., Kankelborg, C.C., Martens, P., \& Warren, H.P., 2000, ApJ, 535, 1047

Torsti, J., Mäkelä, P., Teittinen, M., \& Laivola, J., 2000, ApJ, 544, 1169

Torsti, J., Kocharov, L., Innes, D. E., Laivola, J., \& Sahla, T., 2001, A\&A, 365, 198

Thompson, B. J., Cliver, E. W., Nitta, N., Delannée, C., \& Delaboudinière, J. P., 2000a, Geophys. Res. Lett., 27, 1431

Thompson, B. J., Reynolds, B., Aurass, H., Gopalswamy, N., Gurman, J. B., Hudson, H. S., Martin, S. F., \& St. Cyr, O. C., 2000b, Solar Phys., 193, 161

Vrsnak, B., 2001, J. Geophys. Res., 106, 25249

Wang, H., Goode, P. R., Denker, C., Yang, G., Yurchishin, V., Nitta, N., Gurman, J. B., St. Cyr, C., \& Kosovichev, A. G., 2000, ApJ, 536, 971

Wang, Y.-M. \& Sheeley, Jr, N. R., 2002, ApJ, 567, 1211

Warmuth, A., Vrsnak, B., Aurass, H., \& Hanslmeier, A., 2001, ApJ, 560, L105

Webb, D. F., Lepping, R. P., Burlaga, L. F., Deforest, C. E., Larson, D. E., Martin, S. F., Plunkett, S. P., \& Rust, D. M., 2000, J. Geophys. Res., 105, 27251

Webb, D. F., 2000, JATP, 62, 1415

Wilhelm, K., Dammasch, I.E., Marsch, E., \& Hassler, D.M., 2000, A\&A, 353, 749

Winebarger, A.R., Emslie, A.G., Mariska, J.T., \& Warren, H.P., 2002a, ApJ, 565, 1298

Winebarger, A.R., Updike, A.C., \& Reeves, K.K., 2002b, ApJ, 570, L105

Wood, B. E., Linsky, J. L., Müller, H., \& Zank, G. P., 2001, ApJ, 547, L49

Wu, S. T., Zheng, Huinan, Wang, S., Thompson, B. J., Plunkett, S. P., Zhao, X. P., \& Dryer, M., 2001, J. Geophys. Res., 106, 25089

Zhang, J., Dere, K. P., Howard, R. A., Kundu, M. R., \& White, S. M., 2001, ApJ, 559, 452 